\title{
Asymptotic behaviour of the distribution density of the fractional Lévy motion
}

\author{
ViCTORIA KnOPOVA* and Alexei KuliK**
}

\begin{abstract}
We investigate the distribution properties of the fractional Lévy motion defined by the Mandelbrot-Van Ness representation

$$
Z_{t}^{H}:=\int_{\mathbb{R}} f(t, s) d Z_{s}
$$

where $Z_{s}, s \in \mathbb{R}$, is a (two-sided) real-valued Lévy process, and

$$
f(t, s):=\frac{1}{\Gamma(H+1 / 2)}\left[(t-s)_{+}^{H-1 / 2}-(-s)_{+}^{H-1 / 2}\right], \quad t, s \in \mathbb{R} .
$$

We consider separately the cases $0<H<1 / 2$ (short memory) and $1 / 2<H<$ 1 (long memory), where $H$ is the Hurst parameter, and present the asymptotic behaviour of the distribution density of the process. Some examples are provided, in which it is shown that the behaviour of the density in the cases $0<H<1 / 2$ and $1 / 2<H<1$ is completely different.
\end{abstract}

\section{Introduction}

In this paper we consider the distribution properties of the fractional Lévy motion (FLM, in the sequel). Various versions of the FLM have been used in a number of recent publications in order to interpret some experimental data. Apart from the rigorous mathematical definition, some modifications of the FLM are derived from the physical point of view, see, example, [15], [7], [11]. The FLM driven by an $\alpha$-stable Lévy process is used as a model for describing sub-diffusive effects in physic and biology (see [6], [27]), signal and traffic modeling [20], [19], [10], finance [5], geophysics [22], [26], [13]. We refer to [26] for the discussion in which type of problems the FLM gives an adequate description for the observed phenomena. In the papers quoted above it was shown that the respective phenomena existing in nature can be better described by models, containing the FLM rather than the fractional Brownian motion (FBM). Finally, we refer to [8] for simulations of the FLM, which can be convenient in practical problems.

\footnotetext{
*V.M. Glushkov Institute of Cybernetics, NAS of Ukraine, 40, Acad. Glushkov Ave., 03187, Kiev, Ukraine, vic_knopova@gmx.de

**Institute of Mathematics, NAS of Ukraine, 3, Tereshchenkivska str., 01601 Kiev, Ukraine, kulik@imath.kiev.ua
} 
Similarly to the FBM, the FLM can be defined in two different ways: via the Mandelbrot-Van Ness representation (see [2] and [21]), or via the Molchanov-Golosov representation (see [3]). We also refer to [24] for a bit different definition of the fractional stable motion. These two representations, being equivalent in the Gaussian setting, in the Lévy setting lead, in general, to different processes; see [25]. Note that, in contrast to the FBM, in some cases the FLM can even be a semi-martingale ([1], [4]).

In this paper we focus on the FLM $Z_{t}^{H}$ defined by the Mandelbrot-Van Ness representation, i.e.

$$
Z_{t}^{H}:=\int_{\mathbb{R}} f(t, s) d Z_{s}
$$

where $Z_{s}, s \in \mathbb{R}$, is a (two-sided) real-valued Lévy process, $H \in(0,1)$ is the Hurst parameter, and

$$
f(t, s):=\frac{1}{\Gamma(H+1 / 2)}\left[(t-s)_{+}^{H-1 / 2}-(-s)_{+}^{H-1 / 2}\right], \quad t, s \in \mathbb{R},
$$

where $x_{+}:=\max (x, 0)$. This definition gives a particularly important representative of the class of so-called moving-average fractional Lévy motions. Since the FLM, according to the survey above, is an adequate model to some phenomena in nature, it would be appropriate to investigate deeply its properties. In particular, knowledge of the distribution properties of the FLM would naturally make it possible to solve various problems related to statistical inference, simulation, etc.

In this paper we concentrate on the asymptotic behaviour of the distribution density of the FLM. In contrast to the FBM case, the study of the distribution density of the FLM is much more complicated. In the recent paper [18] we presented the investigation of the distribution density of a FLM in the following cases: (i) $H=1 / 2$, which means merely $Z_{t}^{H} \equiv Z_{t}$, and (ii) $1 / 2<H<1$, which corresponds to the so-called long memory case, see Definition 1.1 in [21]. Both cases can be treated in a unified way using a general result about the asymptotic behaviour of distribution densities of Lévy driven stochastic integrals with deterministic kernels, see Theorem 2.1 in [18]. Since this theorem requires the respective kernel to be bounded, and the kernel (2) is unbounded when $0<H<1 / 2$, the case (iii) $0<H<1 / 2$ cannot be treated in the same way as in [18], and thus requires a completely different approach, which we present below. Further, we show that there is a substantial difference in the behaviour of the density in the cases (i), (ii) on one hand, and the case (iii) on the other hand, namely, the distribution density in these situations exhibits absolutely different types of the asymptotic behaviour. We also emphasize, that in contrast to the situation studied in [18], for the case $0<H<1 / 2$ we do not require the existence of exponential moments of the tails of the Lévy measure.

Let us outline the rest of the paper. In Section 2 we set the notation and formulate our main results, Theorems 1 and 2. In Section 3 we formulate the general result, Theorem 3, on the asymptotic behaviour of the distribution density of Lévy functionals, on which Theorems 1 and 2 are based on. In Section 4 we give two examples which illustrate the effects that may happen in the "extremely heavy-tailed" case, i.e. when condition (17) (see below) fails. In Appendix we give some supplementary statements: the necessary and sufficient condition for the integral (1) to be well defined, and the condition for the respective distribution to possess a density. 


\section{Settings and the main result}

Let $Z_{t}, t \in \mathbb{R}$, be a real-valued (two-sided) Lévy process with the characteristic exponent $\psi$, which means that $Z$ has stationary independent increments, and the characteristic function of an increment is given by

$$
E e^{i z\left(Z_{t}-Z_{s}\right)}=e^{(t-s) \psi(z)}, \quad t>s .
$$

The characteristic exponent $\psi$ admits the Lévy-Khinchin representation

$$
\psi(z)=i a z-b z^{2}+\int_{\mathbb{R}}\left(e^{i u z}-1-i z u 1_{\{|u| \leq 1\}}\right) \mu(d u)
$$

where $a \in \mathbb{R}, b \geq 0$, and $\mu(\cdot)$ is a Lévy measure, i.e. $\int_{\mathbb{R}}\left(1 \wedge u^{2}\right) \mu(d u)<\infty$. To exclude the trivial cases, we assume that $b=0$ and $\mu(\mathbb{R})>0$; that is, $Z$ does not contain a diffusion part, and contains a non-trivial jump part. To simplify the notation we also assume without loss of generality that $Z_{0}=0$ and $a=0$.

We define the integral (1) as a limit in probability of the respective integral sums, see [23, Section 2]. When $H \neq 1 / 2$, the necessary and sufficient condition for this integral to be well defined is

$$
\int_{|u| \geq 1}|u|^{2 /(3-2 H)} \mu(d u)<\infty
$$

see Proposition 7 in Appendix I. Furthermore, it will be shown in Proposition 8 (see Appendix II) that under the same conditions and our standing assumption

$$
\mu(\mathbb{R})>0,
$$

the integral (1) possesses for any $t \neq 0$ a distribution density, which we denote by $p_{t}(x)$, and, moreover, $p_{t} \in C_{b}^{\infty}(\mathbb{R})$. Note that in the Lévy case, i.e. for $H=1 / 2$, available sufficient conditions for the existence of the density $p_{t} \in C_{b}^{\infty}(\mathbb{R})$ are much stronger, see, for example, [14] for the Hartman-Wintner condition.

An important feature of the process $Z_{t}^{H}$ is that one can explicitly write its characteristic function $\phi(t, z):=E e^{i z Z_{t}^{H}}$ (cf. [23, Theorem 2.7]):

$$
\phi(t, z)=e^{\Psi(t,-z)}
$$

where

$$
\Psi(t, z)=\int_{\mathbb{R}} \int_{\mathbb{R}}\left(e^{-i z f(t, s) u}-1+i z f(t, s) u 1_{|u| \leq 1}\right) \mu(d u) d s, \quad z \in \mathbb{R}, \quad t>0 .
$$

Observe, that if the measure $\mu$ possesses exponential moments, the function $\Psi(t, z)$ can be extended with respect to $z$ to the complex plane. Moreover, one can see (cf. Section 3.3) under the assumptions that $\mu\left(\mathbb{R}_{+}\right)>0$, the function

$$
H(x, z):=i z x+\Psi(1, z)
$$

has a unique critical point $i \xi(x)$ on the line $i \mathbb{R}$. Put

$$
D(x):=H(x, i \xi(x)), \quad K(x):=\left.\frac{\partial^{2}}{\partial \xi^{2}} H(x, i \xi)\right|_{\xi=\xi(x)},
$$


and

$$
M_{k}(\xi):=\int_{\mathbb{R}} u^{k} e^{\xi u} \mu(d u), \quad k \geq 2, \quad \xi \in \mathbb{R} .
$$

Fix $t_{0}>0$. In what follows, we write $f \ll g$, if $f / g \rightarrow 0$, and $f \sim g$, if $f / g \rightarrow 1$.

Theorem 1. Let $Z_{t}^{H}, 1 / 2<H<1, t \geq t_{0}$, be a FLM defined by (1), where $Z_{t}$ is a Lévy process with the associate Lévy measure $\mu$. Suppose that the conditions below hold true:

1) $\mu\left(\mathbb{R}^{+}\right)>0$;

2) for all $C \in \mathbb{R}$

$$
\int_{|y| \geq 1} e^{C y} \mu(d y)<\infty
$$

3) $\exists \gamma \in(0,1)$ such that $M_{4}(\xi) \ll M_{2}^{2}(\gamma \xi)$ as $\xi \rightarrow \infty$;

4) $\ln \left(\frac{M_{4}(\xi)}{M_{2}(\xi)} \vee 1\right)+\ln \ln M_{2}(\xi) \ll \xi, \xi \rightarrow+\infty$.

Then the distribution density $p_{t}(x)$ of $Z_{t}^{H}$ exists, $p_{t} \in C_{b}^{\infty}$, and satisfies the asymptotic relation

$$
p_{t}(x) \sim \frac{1}{\sqrt{2 \pi t^{2 H} K\left(x t^{-H-1 / 2}\right)}} e^{t D\left(x t^{-H-1 / 2}\right)}, \quad t+x \rightarrow \infty, \quad(t, x) \in\left[t_{0}, \infty\right) \times \mathbb{R}_{+},
$$

where $D$ and $K$ are defined in (9).

To formulate the result for $0<H<1 / 2$ we need a bit more notation. Let $f(s):=$ $f(1, s)$, see $(2)$ for the definition of $f(t, s)$. Observe that $f(s)$ is monotone decreasing on $(-\infty, 0)$, monotone increasing on $(0,1]$, and the range of $f$ restricted, respectively, to $(-\infty, 0)$ and $(0,1]$, is $(-\infty, 0)$ and $\left[\frac{1}{\Gamma(H+1 / 2)},+\infty\right)$. In addition, the derivative $f^{\prime}(s)$ is well defined and is continuous on $(-\infty, 0)$ and $(0,1)$. Hence, we can put

$$
\ell(y):= \begin{cases}\left(f^{-1}\right)^{\prime}(y)=\frac{1}{f^{\prime}\left(f^{-1}(y)\right)}, & y \in(-\infty, 0) \cup\left[\frac{1}{\Gamma(H+1 / 2)},+\infty\right), \\ 0, & \text { otherwise. }\end{cases}
$$

Note that $\ell(y)$ is non-negative if $y \geq 0$, and is negative otherwise. Define

$$
\mathfrak{m}(r):=\int_{-\infty}^{\infty} \frac{1}{y} \ell\left(\frac{r}{y}\right) \mu(d y), \quad r>0
$$

Recall that (see Definition 4 in [17]) a function $g: \mathbb{R}_{+} \rightarrow \mathbb{R}_{+}$belongs to the class $\mathcal{L} d$ of sub-exponential densities, if $g(x)>0$ for large enough positive $x$, and

$$
\lim _{x \rightarrow+\infty} \frac{(g * g)(x)}{g(x)}=2, \quad \text { and } \quad \lim _{x \rightarrow+\infty} \frac{g(x-y)}{g(x)}=1 \text { for any } y \in \mathbb{R},
$$

where $*$ is a usual definition for the convolution. Fix $t>0$.

Theorem 2. Let $Z_{t}^{H}, 0<H<1 / 2$, be a FLM defined by (1), where $Z_{t}$ is a Lévy process with the associate Lévy measure $\mu$. Suppose that (5) holds true and

$$
\mu\left(\mathbb{R}_{-}\right)>0
$$


Then for every $t>0$ the value of FLM $Z_{t}^{H}$ defined by (1) possesses a probability density $p_{t} \in C_{b}^{\infty}(\mathbb{R})$, which satisfies the following:

i) if

$$
\int_{|u| \geq 1}|u|^{2 /(1-2 H)} \mu(d u)=\infty
$$

and $\mathfrak{m} \in \mathcal{L} d$, then for all $t>0$

$$
p_{t}(x) \sim t^{3 / 2-H} \mathfrak{m}\left(t^{1 / 2-H} x\right), \quad x \rightarrow+\infty ;
$$

ii) if

$$
\int_{|u| \geq 1}|u|^{2 /(1-2 H)} \mu(d u)<\infty
$$

then

$$
p_{t}(x) \sim c_{H}\left(\int_{\mathbb{R}}|u|^{2 /(1-2 H)} \mu(d u)\right) x^{-(3-2 H) /(1-2 H)}, \quad x \rightarrow+\infty,
$$

where

$$
c_{H}=\frac{2}{1-2 H}(\Gamma(H+1 / 2))^{-2 /(1-2 H)} .
$$

Remark 1. a) Apparently, relation (16) holds true when both $x$ and $t$ tend to $+\infty$ in such a way that $x t^{-H-1 / 2} \rightarrow+\infty$. To prove such an extension of Theorem 2 , one should have an extension of [17, Theorem 3.2] which applies to a family of random sums with the variable distribution of the number of summands.

b) Clearly, results similar to (16) and (18) can be formulated for $x \rightarrow-\infty$. In that case one should assume $\mu\left(\mathbb{R}_{+}\right)>0$ instead of $\mu\left(\mathbb{R}_{-}\right)>0$.

To illustrate the crucial difference between the cases treated in Theorem 1 and Theorem 2, consider two particular examples from [18] which concern the case $H>1 / 2$. First, let the Lévy measure $\mu$ of the Lévy noise $Z_{t}$ in (1) be supported in a bounded set. Then (see in [18, Corollary 5.1 and Corollary 5.2]) there exists a constant $c_{*}(\mu)$, defined in terms of the Lévy measure $\mu$ only, such that for any constants $c_{1}>c_{*}(\mu)$ and $c_{2}<c_{*}(\mu)$ there exists $y\left(c_{1}, c_{2}\right)$ such that for $x>y\left(c_{1}, c_{2}\right) t^{H+1 / 2}$ we have

$$
p_{t}(x)\left\{\begin{array}{l}
\geq \exp \left(-\frac{c_{1} x}{\Gamma(H+1 / 2) t^{H-1 / 2}} \ln \left(\frac{x}{t^{H+1 / 2}}\right)\right), \\
\leq \exp \left(-\frac{c_{2} x}{\Gamma(H+1 / 2) t^{H-1 / 2}} \ln \left(\frac{x}{t^{H+1 / 2}}\right)\right) .
\end{array}\right.
$$

Similar statement is available as well when the tails of the Lévy measure admit the following super-exponential estimates: for $u$ large enough

$$
\frac{1}{Q(u)} e^{-b u^{\beta}} \leq \mu([u,+\infty)) \leq Q(u) e^{-b u^{\beta}},
$$

where $b>0$ and $\beta>1$ are some constants, and $Q$ is some polynomial. In this case, instead of (20) we have for any constants $c_{1}>c_{*}(\mu)$ and $c_{2}<c_{*}(\mu)$

$$
p_{t}(x)\left\{\begin{array}{l}
\geq \exp \left(-\frac{c_{1} x}{\Gamma(H+1 / 2) t^{H-1 / 2}} \ln ^{\frac{\beta-1}{\beta}}\left(\frac{x}{t^{H+1 / 2}}\right)\right) \\
\leq \exp \left(-\frac{c_{2} x}{\Gamma(H+1 / 2) t^{H-1 / 2}} \ln ^{\frac{\beta-1}{\beta}}\left(\frac{x}{t^{H+1 / 2}}\right)\right),
\end{array}\right.
$$


for $x>y\left(c_{1}, c_{2}\right) t^{H+1 / 2}$ (again, $c_{*}(\mu)$ is defined in terms of the Lévy measure $\mu$ only).

Comparing (20) and (22), we see that the asymptotic behaviour of the tails of the Lévy measure $\mu$ is substantially involved in the estimates for $p_{t}(x)$. The case $0<H<$ $1 / 2$ is completely different. In particular, if (17) holds true, then $p_{t}(x)$ satisfies (18), where the right-hand side is even independent of $t$, which is an interesting and quite an unexpected fact. We also emphasize that under (17) the polynomial "shape" of the expression in the right-hand side of (18) does not depend on $\mu$, and the only impact of $\mu$ is represented by the multiplier $\int_{\mathbb{R}}|u|^{2 /(1-2 H)} \mu(d u)$. This means that in the case $0<H<1 / 2$ the asymptotic behaviour of $p_{t}(x)$ "mostly" does not depend on $\mu$. However, when $\mu$ is "extremely heavy-tailed", there still remains a possibility for the density $p_{t}(x)$ to be more sensitive with respect to both the Lévy measure $\mu$ and the time parameter $t$. The dichotomy between the "regular" case (when (17) holds) and the "extremely heavytailed" case (when (17) fails) is illustrated in Section 4 below. Such a dichotomy can be informally explained by the competition between the impacts of the kernel $f(t, s)$ on one hand, and of the measure $\mu$ on the other hand.

\section{Proofs}

\subsection{General theorem}

Before we proceed to the proofs, we formulate a central analytical result on the behaviour of the inverse Fourier transform for a certain class of functions. This result plays the key role in the proofs of Theorems 1 and 2 .

Let $I \subset \mathbb{R}$ be some interval and $\mathbb{T}$ be some set of parameters. Consider a function $f: \mathbb{T} \times I \rightarrow \mathbb{R}$, a family of subsets $\mathcal{C}(t, s) \subset \mathbb{R}, t \in \mathbb{T}, s \in I$, and a Lévy measure $\mu$ such that

$$
\begin{gathered}
\int_{I} f^{2}(t, s) d s<\infty, \quad t \in \mathbb{T} \\
\int_{I} \int_{\mathfrak{C}(t, s)}\left(|f(t, s) u|^{2} \wedge 1\right) \mu(d u) d s<\infty \\
\int_{I}\left|\int_{\mathfrak{C}(t, s)}\left(f(t, s) u 1_{|f(t, s) u| \leq 1}-f(t, s) u 1_{|u| \leq 1}\right) \mu(d u)\right| d s<\infty .
\end{gathered}
$$

Then the following function is well defined:

$$
\Psi(t, z)=\int_{I} \int_{\mathcal{C}(t, s)}\left(e^{-i z f(t, s) u}-1+i z f(t, s) u 1_{|u| \leq 1}\right) \mu(d u) d s, \quad z \in \mathbb{R} .
$$

Our aim is to investigate the asymptotic behaviour of the function (provided it exists)

$$
q_{t}(x)=(2 \pi)^{-1} \int_{\mathbb{R}} e^{i x z} \phi(t, z) d z=(2 \pi)^{-1} \int_{\mathbb{R}} e^{-i x z+\Psi(t, z)} d z, \quad t>0, \quad x \in \mathbb{R},
$$

as $(t, x)$ tend to infinity in some appropriate regions. Clearly, when $\mathcal{C}(t, s) \equiv \mathbb{R}$, the function $q_{t}(x)$ is nothing else but the distribution density of the Lévy functional

$$
Y_{t}=\int_{I} f(t, s) d Z_{s}
$$


where $Z_{s}$ is the Lévy process associated with measure $\mu$, without a drift and a Gaussian component.

Assume in addition that for some $\lambda>0$ we have

$$
f(t, s) u \leq \lambda, \quad t \in \mathbb{T}, \quad s \in I, \quad u \in \mathcal{C}(t, s) .
$$

Then it can be shown that the function $\Psi(t, \cdot)$ defined in $(26)$ can be extended to the half-plane $\mathbb{C}_{+}:=\{z \in \mathbb{C}: \operatorname{Im} z \geq 0\}$, and respective extension (we denote it by the same letter $\Psi)$ is continuous on $\mathbb{C}_{+}$and analytical in the inner part of this half-plane.

Consider the function

$$
H(t, x, z):=i z x+\Psi(t, z), \quad z \in \mathbb{C}_{+},
$$

and observe that

$$
\frac{\partial}{\partial \xi} H(t, x, i \xi)=-x+\int_{I} \int_{\mathfrak{C}(t, s)} u f(t, s)\left(e^{\xi f(t, s) u}-1_{|u| \leq 1}\right) \mu(d u) d s \rightarrow \infty, \quad \xi \rightarrow+\infty,
$$

provided that

$$
(\mu \times \operatorname{Leb})(\{(u, s): f(t, s) u>0\})>0 .
$$

Furthermore, under the same condition $\frac{\partial^{2}}{\partial \xi^{2}} H(t, x, i \xi)>0$ for all $\xi \in \mathbb{R}$. Hence,

$$
(0, \infty) \ni \xi \mapsto \frac{\partial}{\partial \xi} H(t, x, i \xi)
$$

is a continuous strictly increasing function with the range $\left(x_{t}-x, \infty\right)$, where

$$
x_{t}=\lim _{\xi \rightarrow 0+} \Psi(t, i \xi)=\int_{I} \int_{\mathfrak{C}(t, s)} u f(t, s) 1_{|u|>1} \mu(d u) d s .
$$

Note that by the above conditions on $f, \mu$ and $\mathcal{C}(t, s)$ the value $x_{t}$ may equal $-\infty$, but is less than $+\infty$. Then for any $x>x_{t}$ there exists unique solution $\xi(t, x)$ to the equation

$$
\frac{\partial}{\partial \xi} H(t, x, i \xi)=0
$$

To formulate the result we need some extra notation:

$$
\begin{gathered}
\mathcal{M}_{k}(t, \xi):=\frac{\partial^{k}}{\partial \xi^{k}} \Psi(t, i \xi), \quad k \geq 1, \\
\mathcal{D}(t, x):=H(t, x, i \xi(t, x)), \quad \mathcal{K}(t, x):=\mathcal{M}_{2}(t, \xi(t, x)),
\end{gathered}
$$

and

$$
\Theta(t, z, B):=\int_{I} \int_{\{u: f(t, s) u \in B, u \in \mathcal{C}(t, s)\}}(1-\cos (f(t, s) z u)) \mu(d u) d s .
$$

Consider a set $\mathcal{A} \subset\left\{(t, x): t \in \mathbb{T}, x>x_{t}\right\} \subset \mathbb{T} \times \mathbb{R}$, and define

$$
\mathcal{T}:=\left\{t: \exists x \in\left(x_{t}, \infty\right),(t, x) \in \mathcal{A}\right\}, \quad \mathcal{B}:=\{(t, \xi): \exists(t, x) \in \mathcal{A},(t, \xi)=(t, \xi(t, x))\} .
$$

Finally, suppose that the function $\theta: \mathcal{T} \rightarrow(0,+\infty)$ is bounded away from zero on $\mathbb{T}$, and the function $\chi: \mathcal{T} \rightarrow(0,+\infty)$ is bounded away from zero on every set $\{t: \theta(t) \leq c\}$, $c>0$. 
Theorem 3. Assume the following.

H1 Conditions (23) - (25), (29), and (31) hold true.

H2 $\mathcal{M}_{4}(t, \xi) \ll \mathcal{M}_{2}^{2}(t, \xi), \theta(t)+\xi \rightarrow \infty,(t, \xi) \in \mathcal{B}$.

H3 For $\theta(t)+\xi \rightarrow \infty,(t, \xi) \in \mathcal{B}$.

$$
\begin{aligned}
\ln \left(\left(\chi^{-2}(t) \frac{\mathcal{M}_{4}(t, \xi)}{\mathcal{M}_{2}(t, \xi)}\right) \vee 1\right) & +\ln \left(\left(\ln \left(\left(1 \vee \chi^{-1}(t)\right) \mathcal{M}_{2}(t, \xi)\right)\right) \vee 1\right) \\
& \ll \ln \theta(t)+\chi(t) \xi
\end{aligned}
$$

H4 There exist $R>0$ and $\delta>0$ such that

$$
\Theta\left(t, z, \mathbb{R}_{+}\right) \geq(1+\delta) \ln (\chi(t)|z|), \quad t \in \mathcal{T}, \quad|z|>R .
$$

H5 There exists $r>0$ such that for every $\epsilon>0$,

$$
\inf _{|z|>\epsilon} \Theta(t, z,[r \chi(t),+\infty)) \geq c \theta(t)\left((\epsilon \chi(t))^{2} \wedge 1\right), \quad t \in \mathcal{T}, \quad c>0 .
$$

Then the function $q_{t}(x)$ given by (27) is well-defined, and satisfies

$$
q_{t}(x) \sim \frac{1}{\sqrt{2 \pi \mathcal{K}(t, x)}} e^{\mathcal{D}(t, x)}, \quad \theta(t)+x \rightarrow \infty, \quad(t, x) \in \mathcal{A} .
$$

Up to some straightforward and purely technical modifications, the proof of Theorem 3 coincides with the proof of [18, Theorem 2.1], and therefore is omitted. Here we only remark that the proof is based on an appropriate modification of the saddle point method, see [9] for details.

\subsection{Outline of the proofs}

One can prove Theorem 1 using a simplified version of Theorem 3 with $\mathcal{C}(t, s) \equiv \mathbb{R}$, and the scaling property of the function $f(t, s)$ :

$$
f(t, s)=|t|^{H-1 / 2} f\left(\frac{s}{t}\right)
$$

where

$$
f(s)=f(1, s)=\frac{1}{\Gamma(H+1 / 2)}\left[(1-s)_{+}^{H-1 / 2}-(-s)_{+}^{H-1 / 2}\right], \quad s \in \mathbb{R} ;
$$

see [18] for details.

Let us turn now to the proof of Theorem 2. To make the proof of Theorem 2 more transparent, we first sketch its main idea. In particular, we show how Theorem 3 applies in the situation when the function $f(t, s)$ is unbounded.

According to [23, Theorem 2.7], the characteristic function $\phi(t, z)(\mathrm{cf}$. (7)) can be decomposed for any fixed $\lambda>0$ as

$$
\phi(t, z)=\phi_{1}(t, z) \phi_{2}(t, z)=e^{\psi_{1}(t, z)} e^{\psi_{2}(t, z)},
$$


where

$$
\begin{gathered}
\psi_{1}(t, z):=\int_{-\infty}^{t} \int_{\{u: u f(s / t) \leq \lambda\}}\left(e^{i z f(t, s) u}-1-i z f(t, s) u 1_{|u| \leq 1}\right) \mu(d u) d s, \\
\psi_{2}(t, z):=\int_{-\infty}^{t} \int_{\{u: u f(s / t)>\lambda\}}\left(e^{i z f(t, s) u}-1\right) \mu(d u) d s+i z a(t), \\
a(t):=\int_{-\infty}^{t} \int_{\{u: u f(s / t)>\lambda\}} f(t, s) u 1_{|u| \leq 1} \mu(d u) d s=t^{H+1 / 2} a(1) .
\end{gathered}
$$

In the last identity we have used the scaling property (38) of the kernel $f$.

The function $\left|\phi_{1}(t, z)\right|$ is integrable with respect to $z$ for any $t>0$, see Remark 4 in the Appendix II below. Then there exists the distribution density

$$
\tilde{p}_{t}(x):=\frac{1}{2 \pi} \int_{\mathbb{R}} e^{-i z x} \phi_{1}(t, z) d z,
$$

and thus the required density $p_{t}(x)$ can be written as the convolution

$$
p_{t}(x)=\left(\tilde{p}_{t} * P_{t}\right)(x)
$$

where $P_{t}(d y)$ is the probability measure corresponding to the characteristic function $\phi_{2}(t, z)$. Define the measure $M_{t}(d y)$ by the relation

$$
\int_{\mathbb{R}} g(y) M_{t}(d y)=\int_{-\infty}^{t} \int_{\{u: u f(s / t)>\lambda\}} g(f(t, s) u) \mu(d u) d s,
$$

where $g$ is an arbitrary measurable and bounded function. Then, up to the shift by $a(t)$, the measure $P_{t}(d y)$ is equal to the distribution of the compound Poisson random variable with the intensity of the Poisson part equal to $M_{t}(d y)$. In other words,

$$
P_{t}(d y)=\delta_{a(t)}(d y) *\left(e^{-M_{t}(\mathbb{R})} \delta_{0}(d y)+e^{-M_{t}(\mathbb{R})} \sum_{k=1}^{\infty} \frac{1}{k !} M_{t}^{* k}(d y)\right),
$$

where $M_{t}^{* k}(d y)$ is the $k$-fold convolution of $M_{t}(d y)$.

It follows from the scaling property $(38)$ that $M_{t}(\mathbb{R})=t \Lambda$ with

$$
\Lambda=\int_{-\infty}^{1} \int_{\{u: u f(s)>\lambda\}} \mu(d u) .
$$

Furthermore, we prove that the measure $M_{t}(d y)$ is absolutely continuous with respect to the Lebesgue measure with the density

$$
m_{t}(x)=t^{3 / 2-H} \mathfrak{m}\left(t^{1 / 2-H} x\right) 1_{\left\{x>\lambda t^{H-1 / 2}\right\}},
$$

where the function $\mathfrak{m}$ is defined by (13). Then (43) can be written in the form

$$
p_{t}(x)=e^{-\Lambda t} \tilde{p}_{t}(x-a(t))+\int_{\mathbb{R}} \rho_{t}(x-y) \tilde{p}_{t}(y-a(t)) d y,
$$


where

$$
\rho_{t}(x):=e^{-t \Lambda} \sum_{k=1}^{\infty} \frac{m_{t}^{* k}(x)}{k !} .
$$

Clearly, $\rho_{t}$ is the density of a random sum with the distribution of one term represented by $m_{t}$. Suppose that the function $\mathfrak{m}$ is sub-exponential. Then it follows from $[17$, Theorem 3.2] that the density $\rho_{t}$ is sub-exponential as well, and

$$
\rho_{t}(x) \sim\left(e^{-t \Lambda} \sum_{k=1}^{\infty} \frac{k}{k !}(t \Lambda)^{k-1}\right) m_{t}(x)=m_{t}(x), \quad x \rightarrow+\infty,
$$

where we used that $\int_{0}^{\infty} m_{t}(x) d x=M_{t}(\mathbb{R})=t \Lambda$.

To estimate $\tilde{p}_{t}(x)$ we apply Theorem 3 . Namely, in Proposition 4 below we show that for a given $\varepsilon>0$ there exists $y(\varepsilon)>0$ such that

$$
\begin{array}{r}
\exp \left(-\frac{(1+\varepsilon) x}{\lambda t^{H-1 / 2}} \ln \frac{x}{t^{H+1 / 2}}\right) \leq \tilde{p}_{t}(x) \leq \exp \left(-\frac{(1-\varepsilon) x}{\lambda t^{H-1 / 2}} \ln \frac{x}{t^{H+1 / 2}}\right) \\
x t^{-H-1 / 2} \geq y(\varepsilon) .
\end{array}
$$

Since a sub-exponential function decays slower than any exponential function (cf. [17]), the term $m_{t}$ dominates both $\tilde{p}_{t}(x)$ and the integral term in (48). In such a way, (48), (50) and (51) provide the required relation (16).

Let us summarize the idea explained above. The distribution of $Z_{t}^{H}$ is decomposed in two parts. For one part, the distribution density is controlled by means of the respective version of the saddle point method, while for the other part the distribution can be evaluated in the form of the series of convolution powers with the explicitly given law of the first summand. Similarly to Theorem 2.1 in [18], Theorem 3 provides a flexible version of the saddle point method, which is applicable to a wide variety of integrals of the form (27). Thus one can expect that the approach presented above can be extended to other processes of the form (1) with unbounded kernels $f(t, s)$. To keep the exposition reasonably tight, in this paper we do not investigate this possibility in the whole generality, and restrict our considerations to the important particular case of the FLM with $0<H<1 / 2$.

\subsection{Properties of $\tilde{p}_{t}(x)$}

Proposition 4. Under (15), for a given $\varepsilon>0$ there exists $y(\varepsilon)>0$ such that $\tilde{p}_{t}(x)$ satisfies (51).

Remark 2. Note that in the above Proposition we do not assume that $t>0$ is fixed.

Proof. We use Theorem 3 with $\theta(t)=t, \chi(t)=t^{H-\frac{1}{2}}, \mathbb{T}=\left[t_{0}, \infty\right), I=(-\infty, t], \mathcal{C}(t, s)=$ $\{u: f(t, s) u \leq \lambda\}$, and $\mathcal{A}=\left\{(t, x) \subset\left[t_{0}, \infty\right) \times \mathbb{R}_{+}: x t^{-H-1 / 2} \geq c\right\}$. Here $t_{0}, c$ and $\lambda$ are some positive constants. Then condition H1 is satisfied: (29) holds true by the construction, (31) holds true thanks to (15), and (23) - (25) can be proved using the same estimates as in the proof of Proposition 7 in Appendix I (we omit the details). 
Recall that $f$ possesses the self-similarity property (38). Then

$$
\mathcal{M}_{k}(t, \zeta)=\chi^{k}(t) t \mathcal{M}_{k}(\chi(t) \zeta),
$$

where

$$
\mathcal{M}_{k}(\zeta):=\frac{\partial^{k}}{\partial \zeta^{k}} H(1, x, i \zeta(1, x))= \begin{cases}\int_{-\infty}^{\lambda} u\left(e^{u \zeta}-1\right) N(d u), & k=1, \\ \int_{-\infty}^{\lambda} u^{k} e^{u \zeta} N(d u), & k \geq 2 .\end{cases}
$$

Here $N(d u):=\int_{-\infty}^{1} \tilde{\mu}_{s}(d u) d s$, and $\tilde{\mu}_{s}(d u)$ is the image measure of $\mu(d u)$ under the mapping $u \mapsto f(s) u$. The choice of $\lambda$ above can be made in such a way that every segment $(\lambda-\varepsilon, \lambda)$ has a positive measure $N$. Then it can be shown (e.g., [18, Example 3.1]) that for any $\varepsilon>0$

$$
e^{\zeta(\lambda-\varepsilon)} \ll \mathcal{M}_{k}(\zeta), \quad \mathcal{M}_{k}(\zeta)-\lambda^{k} N(\{\lambda\}) e^{\zeta \lambda} \ll e^{\zeta \lambda}, \quad \zeta \rightarrow+\infty .
$$

Moreover, applying the Laplace method we get

$$
\mathcal{M}_{k}(\zeta) \sim \lambda^{k} \mathcal{M}_{0}(\zeta), \quad \zeta \rightarrow \infty
$$

where

$$
\mathcal{M}_{0}(\zeta)=\int_{-\infty}^{\lambda}\left(e^{\zeta u}-1-\zeta u\right) N(d u)
$$

Note that the solution $\xi(t, x)$ to (33) satisfies

$$
\xi(t, x)=\chi^{-1}(t) \zeta\left(x t^{-H-1 / 2}\right), \quad \text { where } \quad \zeta(x):=\xi(1, x) .
$$

Since $\zeta(x)$ is the solution to $\mathcal{M}_{1}(\zeta(x))=x$, we have $\zeta(x) \rightarrow \infty$ as $x \rightarrow \infty$. Then by (55) and the definition of $\mathcal{A}$ we have $\chi(t) \xi \rightarrow \infty$ as $t+\xi \rightarrow \infty,(t, \xi) \in \mathcal{B}$, implying

$$
\mathcal{M}_{0}(\chi(t) \xi)>0, \quad \text { as } t+\xi \rightarrow \infty,(t, \xi) \in \mathcal{B} \text {. }
$$

Therefore, by (52) and (54) we have H2:

$$
\frac{\mathcal{M}_{4}(t, \xi)}{\mathcal{M}_{2}^{2}(t, \xi)} \sim \frac{1}{t \mathcal{M}_{0}(\chi(t) \xi)} \ll 1, \quad t+\xi \rightarrow \infty, \quad(t, \xi) \in \mathcal{B} .
$$

Analogously, we have

$$
\begin{gathered}
\chi^{-2}(t) \frac{\mathcal{M}_{4}(t, \xi)}{\mathcal{M}_{2}(t, \xi)} \sim \lambda^{2}, \\
\ln \left(\left(\ln \left(\left(1 \vee \chi^{-1}(t)\right) \mathcal{M}_{2}(t, \xi)\right)\right) \vee 1\right) \leq C(\ln \ln t+\ln (\chi(t) \xi)) \ll \ln t+\chi(t) \xi,
\end{gathered}
$$

as $t+\xi \rightarrow \infty,(t, \xi) \in \mathcal{B}$, which provides $\mathbf{H 3}$.

To show H4, take $b<0$ and $\varepsilon>0$ such that $0<\mu([\lambda / f(b),-\varepsilon])<\infty$. Then by (74) (see Appendix II) we have for $|z| \geq R$ with some $R$ large enough

$$
\begin{aligned}
\Theta\left(t, z, \mathbb{R}_{+}\right) & \geq t \int_{-\infty}^{0} \int_{\{u: 0<f(s) u \leq \lambda\}}(1-\cos (\chi(t) z u f(s))) \mu(d u) d s \\
& \geq t_{0} \int_{-\infty}^{b} \int_{\lambda / f(b)}^{-\varepsilon}(1-\cos (\chi(t) z u f(s))) \mu(d u) d s \\
& \geq c t_{0} \mu([\lambda / f(b),-\varepsilon]) \ln (\chi(t) \varepsilon|z|) .
\end{aligned}
$$


Since we can chose in (74) $c>0$ arbitrary large, condition $\mathbf{H 4}$ holds true. Finally, estimate (72) (see Appendix II) provides H5: since $\mu\left(\mathbb{R}_{-}\right)>0$, there exists $(a, b) \subset$ $(-\infty, 0), q>0$, such that $0<\mu([q \lambda / f(b), \lambda / f(b)])<\infty$ (note that $f(b)<0)$, and

$$
\begin{aligned}
\inf _{|z| \geq c} \Theta(t, z,[\chi(t) q, \infty)) & \geq t \inf _{|z| \geq c} \int_{a}^{b} \int_{q \lambda / f(s)}^{\lambda / f(s)}(1-\cos (\chi(t) z u f(s))) \mu(d u) d s \\
& \geq t \inf _{|z| \geq c} \int_{q \lambda / f(b)}^{\lambda / f(b)}\left((z \chi(t) u)^{2} \wedge 1\right) \mu(d u) \\
& \geq c_{1} t\left((\chi(t) c)^{2} \wedge 1\right) .
\end{aligned}
$$

Thus, all conditions of Theorem 3 are satisfied, and therefore (37) holds true.

By (53),

$$
\ln \mathcal{M}_{1}(\zeta) \sim \lambda \zeta, \quad \zeta \rightarrow+\infty .
$$

Since $\zeta(x)($ cf. $(55))$ is the solution to $\mathcal{M}_{1}(\zeta(x))=x$, this means that

$$
\zeta(x) \sim \frac{\ln x}{\lambda}, \quad x \rightarrow+\infty .
$$

Denote $\mathcal{D}(x):=H(1, x, i \xi(1, x))$, then using (54) with $k=1,2$ we get from the previous relation that

$$
\mathcal{D}(x) \sim-\frac{x \ln x}{\lambda}, \quad \mathcal{M}_{2}(\zeta(x)) \sim \lambda x, \quad x \rightarrow+\infty .
$$

From (37) and (56) we deduce that for a given $\varepsilon>0$ there exists $y(\varepsilon)>0$ such that $(51)$ holds true.

\subsection{Properties of $M_{t}(d x)$ and the completion of the proof}

Lemma 5. For every $t>0$ we have $M_{t}(d x)=m_{t}(x) d x$ with $m_{t}$ defined by (47).

Proof. Let $g$ be an arbitrary bounded measurable function. Using the scaling property (38) of the kernel $f(t, s)$ we can rewrite (44) as

$$
\begin{aligned}
& \int_{-\infty}^{\infty} g(y) M_{t}(d y)=\int_{-\infty}^{t} \int_{\{y f(s / t)>\lambda\}} g\left(t^{H-1 / 2} f(s / t) y\right) \mu(d y) d s \\
& \quad=t \int_{-\infty}^{0} \int_{\{y<-\lambda / f(s)\}} g_{t}(f(s) y) \mu(d y) d s+t \int_{0}^{1} \int_{\{y>\lambda / f(s)\}} g_{t}(f(s) y) \mu(d y) d s \\
& \quad=: I_{-}+I_{+} .
\end{aligned}
$$

Here $g_{t}(y):=g\left(t^{H-1 / 2} y\right)$, and recall that the function $f(s)=f(1, s)$ is monotone on $(-\infty, 0)$ and $(0,1)$, in particular, $f$ is positive on $(0,1)$ and negative on $(-\infty, 0)$. Let us transform the integrals $I_{+}$and $I_{-}$separately.

Recall that the range of the restriction of $f$ to $(0,1)$ equals $\left[\frac{1}{\Gamma(H+1 / 2)},+\infty\right)$. Then, making the change of variables $\tau=1 / f(s)$, we get

$$
\begin{aligned}
I_{+} & =t \int_{0}^{\Gamma(H+1 / 2)}\left(\int_{\{y>\lambda \tau\}} g_{t}\left(\frac{y}{\tau}\right) \mu(d y)\right) \frac{d \tau}{\tau^{2} f^{\prime}\left(f^{-1}\left(\frac{1}{\tau}\right)\right)} \\
& =t \int_{0}^{+\infty}\left(\int_{\{y>\lambda \tau\}} g_{t}\left(\frac{y}{\tau}\right) \mu(d y)\right) \ell\left(\frac{1}{\tau}\right) \frac{d \tau}{\tau^{2}} .
\end{aligned}
$$


In the second identity we take into account that, according to (12), the function $\ell$ vanishes on $\left(0, \frac{1}{\Gamma(H+1 / 2)}\right)$. Then the further change of variables $r=y / \tau$ and the Fubini theorem give

$$
I_{+}=t \int_{\lambda}^{\infty} g_{t}(r)\left(\int_{0}^{\infty} \frac{1}{y} \ell\left(\frac{r}{y}\right) \mu(d y)\right) d r .
$$

Performing similar calculations, we get

$$
I_{-}=t \int_{\lambda}^{\infty} g_{t}(r)\left(\int_{-\infty}^{0} \frac{1}{y} \ell\left(\frac{r}{y}\right) \mu(d y)\right) d r .
$$

Adding the expressions for $I_{+}$and $I_{-}$we get

$$
\int_{-\infty}^{\infty} g(y) M_{t}(d y)=t \int_{\lambda}^{\infty} g\left(t^{H-1 / 2} r\right) \mathfrak{m}(r) d r=\int_{\lambda t^{H-1 / 2}} g(y)\left[t^{3 / 2-H} \mathfrak{m}\left(t^{1 / 2-H} y\right)\right] d y .
$$

Let us summarize: we have (48) and (51); in addition, if $\mathfrak{m}$ is sub-exponential, we have (50) by Theorem 3.2 in [17]. In such a way we obtain the proof of part i) of Theorem 2. The following lemma completes the proof of the statement ii).

Lemma 6. If $\mu$ satisfies (17), then

$$
\mathfrak{m}(r) \sim c_{H}\left(\int_{\mathbb{R}}|u|^{2 /(1-2 H)} \mu(d u)\right) r^{-(3-2 H) /(1-2 H)}, \quad r \rightarrow \infty,
$$

where the constant $c_{H}$ is defined in (19). In particular, $\mathfrak{m} \in \mathcal{L} d$.

Proof. Write $\mathfrak{m}=\mathfrak{m}_{-}+\mathfrak{m}_{+}$, where

$$
\mathfrak{m}_{-}(r):=\int_{-\infty}^{0} \frac{1}{y} \ell\left(\frac{r}{y}\right) \mu(d y), \quad \mathfrak{m}_{+}(r):=\int_{0}^{+\infty} \frac{1}{y} \ell\left(\frac{r}{y}\right) \mu(d y) .
$$

On the positive half-axis the function $\ell$ can be calculated explicitly:

$$
\ell(y)=c_{H} y^{-(3-2 H) /(1-2 H)} 1_{\{y \geq 1 / \Gamma(H+1 / 2)\}},
$$

where $c_{H}$ is given by (19). Then

$$
\begin{aligned}
\mathfrak{m}_{+}(r) & =c_{H} r^{-(3-2 H) /(1-2 H)} \int_{0}^{r \Gamma(H+1 / 2)} y^{2 /(1-2 H)} \mu(d y) \\
& \sim c_{H}\left(\int_{0}^{+\infty} y^{2 /(1-2 H)} \mu(d y)\right) r^{-(3-2 H) /(1-2 H)}, \quad r \rightarrow+\infty .
\end{aligned}
$$

Note that $2 /(1-2 H)>2$ and $\mu$ is a Lévy measure (that is, $\int_{|y| \leq 1} y^{2} \mu(d y)<\infty$ ), which together with (17) implies that $\int_{0}^{+\infty} y^{2 /(1-2 H)} \mu(d y)<\infty$.

On the negative half-axis one has

$$
\ell(y) \sim \begin{cases}-c_{H}(-y)^{-(3-2 H) /(1-2 H)}, & y \rightarrow-\infty \\ -\hat{c}_{H}(-y)^{-(5-2 H) /(3-2 H)}, & y \rightarrow 0-\end{cases}
$$


with $\hat{c}_{H}=\frac{2}{3-2 H}\left(\frac{1-2 H}{2 \Gamma(H+1 / 2)}\right)^{2 /(3-2 H)}$. Take arbitrary $\varepsilon>0$ and choose $a_{\varepsilon}, b_{\varepsilon}>0$ such that

$$
\begin{gathered}
-\ell(y) \leq\left(\hat{c}_{H}+\varepsilon\right)(-y)^{-(5-2 H) /(3-2 H)}, \quad(-y) \in\left(0, a_{\varepsilon}\right), \\
-\ell(y) \leq\left(c_{H}+\varepsilon\right)(-y)^{-(3-2 H) /(1-2 H)}, \quad(-y)>b_{\varepsilon} .
\end{gathered}
$$

Then

$$
\begin{aligned}
\mathfrak{m}_{-}(r) & =\left[\int_{-\infty}^{-r / a_{\varepsilon}}+\int_{-r / a_{\varepsilon}}^{-r / b_{\varepsilon}}+\int_{-r / b_{\varepsilon}}^{0}\right] \frac{1}{y} \ell\left(\frac{r}{y}\right) \mu(d y) \\
& \leq\left(\hat{c}_{H}+\varepsilon\right) r^{-(5-2 H) /(3-2 H)} \int_{-\infty}^{-r / a_{\varepsilon}}(-y)^{2 /(3-2 H)} \mu(d y) \\
& +\sup _{y \in\left[-b_{\varepsilon},-a_{\varepsilon}\right]}(-\ell(y)) \int_{-r / a_{\varepsilon}}^{-r / b_{\varepsilon}}\left(-\frac{1}{y}\right) \mu(d y) \\
& +\left(c_{H}+\varepsilon\right) r^{-(3-2 H) /(1-2 H)} \int_{-r / b_{\varepsilon}}^{0}(-y)^{2 /(1-2 H)} \mu(d y) \\
& =I_{1}(r)+I_{2}(r)+I_{3}(r) .
\end{aligned}
$$

By condition (17), one has

$$
\begin{aligned}
\int_{-\infty}^{-r / a_{\varepsilon}}(-y)^{2 /(3-2 H)} \mu(d y) & =\int_{-\infty}^{-r / a_{\varepsilon}}(-y)^{2 /(1-2 H)}(-y)^{-4 /((1-2 H)(3-2 H))} \mu(d y) \\
& \leq\left(\frac{a_{\epsilon}}{r}\right)^{4 /((1-2 H)(3-2 H))} \int_{-\infty}^{-r / a_{\varepsilon}}(-y)^{2 /(1-2 H)} \mu(d y) \\
& \leq c_{1} r^{-4 /((1-2 H)(3-2 H))}
\end{aligned}
$$

which implies

$$
r^{(3-2 H) /(1-2 H)} I_{1}(r) \rightarrow 0, \quad r \rightarrow \infty .
$$

Further, since by (17) we have

$$
r^{2 /(1-2 H)} \mu((-\infty,-r]) \leq \int_{-\infty}^{-r}(-y)^{2 /(1-2 H)} \mu(d y) \rightarrow 0, \quad r \rightarrow \infty
$$

then

$$
r^{(3-2 H) /(1-2 H)} I_{2}(r) \leq c_{2} r^{2 /(1-2 H)} \mu\left(\left(-\infty,-r / b_{\varepsilon}\right]\right) \rightarrow 0, \quad r \rightarrow+\infty .
$$

Thus,

$$
\limsup _{r \rightarrow+\infty} r^{(3-2 H) /(1-2 H)} \mathfrak{m}_{-}(r) \leq\left(c_{H}+\varepsilon\right) \int_{-\infty}^{0}(-y)^{2 /(1-2 H)} \mu(d y) .
$$

The same argument provides the desired lower bound for $\lim \inf _{r \rightarrow+\infty}$ with $c_{H}-\varepsilon$ instead of $c_{H}+\varepsilon$. Since $\varepsilon$ is arbitrary, these two estimates lead to the relation

$$
\mathfrak{m}_{-}(r) \sim c_{H}\left(\int_{-\infty}^{0}(-y)^{2 /(1-2 H)} \mu(d y)\right) r^{-(3-2 H) /(1-2 H)}, \quad r \rightarrow+\infty,
$$

which completes the proof. 


\section{Two examples: the "extremely heavy-tailed" case}

In this section we give two examples which illustrate the behaviour of $p_{t}(x)$ when condition (17) fails. In this case we say that the measure $\mu$ is "extremely heavy-tailed".

Example 1. Denote by $\mu_{-}(x)=\mu((-\infty,-x]), \mu_{+}(x)=\mu([x,+\infty)), x>0$, the "tails" of the Lévy measure $\mu$, and assume that $\mu_{-}$and $\mu_{+}$are regularly varying at $+\infty$, that is, there exist $\alpha_{ \pm} \in \mathbb{R}$ and slowly varying functions $L_{ \pm}$, such that

$$
\mu_{ \pm}(x)=x^{-\alpha_{ \pm}} L_{ \pm}(x),
$$

see, for example, [12, Chapter VIII, §8]. We investigate the behaviour of the functions $\mathfrak{m}_{-}$and $\mathfrak{m}_{+}$introduced in the proof of Lemma 6 .

We assume

$$
\alpha_{ \pm} \in\left(\frac{2}{3-2 H}, \frac{2}{1-2 H}\right) .
$$

Note that condition $\alpha_{ \pm} \geq 2 /(3-2 H)$ is necessary for (5) to hold true, and if $\alpha_{ \pm}>2 /(1-$ $2 H$ ) then (17) holds true and the required behaviour of $\mathfrak{m}_{-}$and $\mathfrak{m}_{+}$is already described in Lemma 6. In order to simplify the exposition, we exclude from the consideration the critical values $\alpha_{ \pm}=2 /(3-2 H)$ and $\alpha_{ \pm}=2 /(1-2 H)$.

The asymptotic behaviour of $\mathfrak{m}_{+}$can be obtained almoststraightforwardly using the standard result on the behaviour of the integrals w.r.t. the measures with regularly varying tails, see [12, Chapter VIII, $\S 9$, Theorem 2]:

$$
\mathfrak{m}_{+}(r) \sim\left(\frac{\mu_{+}(r)}{r}\right) \int_{0}^{+\infty} \Phi(z) \mathfrak{p}_{+}(z) d z, \quad r \rightarrow \infty, \quad \text { with } \quad \mathfrak{p}_{+}(z)=\alpha_{+} z^{-\alpha_{+}-1} .
$$

The investigation of the behaviour of $\mathfrak{m}_{-}$is slightly more complicated. However, the argument here is quite standard, and therefore we just sketch it.

Write $\mathfrak{m}_{-}$in the form

$$
\mathfrak{m}_{-}(r)=r^{-1} \int_{-\infty}^{0} \Phi\left(\frac{y}{r}\right) \mu(d y), \quad \Phi(x):=\frac{1}{x} \ell\left(\frac{1}{x}\right) .
$$

It follows from (60) that there exists a constant $C$ such that $\Phi(x) \leq C(-x)^{2 /(1-2 H)}$ for $(-x)$ small enough and $\Phi(x) \leq C(-x)^{2 /(3-2 H)}$ for $(-x)$ large enough. Then, by [12, Chapter VIII, $\S 9$, Theorem 2], (see also Problem 30 in $\S 10$ of the same Chapter) we have for $A$ small enough and $B$ large enough

$$
\begin{aligned}
\int_{-\infty}^{-B r} \Phi\left(\frac{y}{r}\right) \mu(d y) & \leq C r^{-2 /(3-2 H)} \int_{-\infty}^{-B r}(-y)^{2 /(3-2 H)} \mu(d y) \\
& \leq C_{H, \alpha_{-}}^{1} B^{2 /(3-2 H)} \mu_{-}(B r) \\
\int_{-A r}^{0} \Phi\left(\frac{y}{r}\right) \mu(d y) & \leq C r^{-2 /(1-2 H)} \int_{-A r}^{0}(-y)^{2 /(1-2 H)} \mu(d y) \\
& \leq C_{H, \alpha_{-}}^{2} A^{2 /(1-2 H)} \mu_{-}(A r),
\end{aligned}
$$


with some explicitly given constant $C_{H, \alpha_{-}}^{i} \in(0, \infty), i=1,2$. We have

$$
\limsup _{r \rightarrow+\infty} A^{2 /(1-2 H)} \frac{\mu_{-}(A r)}{\mu_{-}(r)}=A^{2 /(1-2 H)-\alpha_{-}} \limsup _{r \rightarrow+\infty} \frac{L_{-}(A r)}{L_{-}(r)}=A^{2 /(1-2 H)-\alpha_{-}}
$$

and, similarly,

$$
\limsup _{r \rightarrow+\infty} B^{2 /(3-2 H)} \frac{\mu_{-}(B r)}{\mu_{-}(r)}=B^{2 /(3-2 H)-\alpha_{-}} .
$$

Then, by condition (61), for every $\varepsilon>0$ one can choose $A$ and $B$ in such a way that

$$
\limsup _{r \rightarrow+\infty} \frac{1}{\mu_{-}(r)}\left(\int_{-\infty}^{0} \Phi\left(\frac{y}{r}\right) \mu(d y)-\int_{-B r}^{-A r} \Phi\left(\frac{y}{r}\right) \mu(d y)\right) \leq \varepsilon .
$$

Further, the function $\Phi$ is continuous and positive on $[-B,-A]$. Therefore, there exists a piece-wise constant function $\Phi_{\varepsilon}$ such that

$$
(1-\varepsilon) \Phi_{\varepsilon} \leq \Phi \leq(1+\varepsilon) \Phi_{\varepsilon}
$$

Clearly, one has for every segment $(a, b] \subset \mathbb{R}_{-}$

$$
\mu((r a, r b]) \sim \mu_{-}(r) \int_{a}^{b} \mathfrak{p}_{-}(z) d z, \quad r \rightarrow \infty, \quad \text { with } \quad \mathfrak{p}_{-}(z)=\alpha_{-}(-z)^{-\alpha_{-}-1} .
$$

Therefore,

$$
\frac{1}{\mu_{-}(r)} \int_{-B r}^{-A r} \Phi_{\varepsilon}\left(\frac{y}{r}\right) \mu(d y) \rightarrow \int_{-B}^{-A} \Phi_{\varepsilon}(z) \mathfrak{p}_{-}(z) d z, \quad r \rightarrow+\infty .
$$

Combined with (63) and (64), this gives

$$
\limsup _{r \rightarrow+\infty} \frac{1}{\mu_{-}(r)} \int_{-\infty}^{0} \Phi\left(\frac{y}{r}\right) \mu(d y) \leq \varepsilon+\frac{1+\varepsilon}{1-\varepsilon} \int_{-\infty}^{0} \Phi(z) \mathfrak{p}_{-}(z) d z
$$

One can write in the same fashion the lower bound for $\lim \inf _{r \rightarrow+\infty}$ (we omit the calculation). Then, since $\varepsilon>0$ is arbitrary, we finally arrive at

$$
\mathfrak{m}_{-}(r) \sim\left(\frac{\mu_{-}(r)}{r}\right) \int_{-\infty}^{0} \Phi(z) \mathfrak{p}_{-}(z) d z, \quad r \rightarrow \infty .
$$

This and (62) give that the function

$$
\mathfrak{m}(r) \sim \frac{1}{r}\left(\mu_{-}(r) \int_{-\infty}^{0} \Phi(z) \mathfrak{p}_{-}(z) d z+\mu_{+}(r) \int_{0}^{+\infty} \Phi(z) \mathfrak{p}_{+}(z) d z\right), \quad r \rightarrow+\infty
$$

clearly belongs to the class $\mathcal{L} d$, and thus the statement i) of Theorem 2 holds true.

Consider, for instance, the " $\alpha$-stable-like" case

$$
\mu_{-}(r) \sim C_{-} r^{-\alpha}, \quad \mu_{+}(r) \sim C_{+} r^{-\alpha}, \quad \text { with } \quad \alpha \in\left(\frac{2}{3-2 H}, \frac{2}{1-2 H}\right) .
$$


Then (16) and the above calculations give

$$
p_{t}(x) \sim t^{1-\alpha(1 / 2-H)} x^{-\alpha-1} \int_{\mathbb{R}} \Phi(z) \mu_{\alpha, C_{-}, C_{+}}(d z), \quad x \rightarrow+\infty
$$

with

$$
\mu_{\alpha, C_{-}, C_{+}}(d z)=\alpha|z|^{-\alpha-1}\left(C_{-} 1_{\mathbb{R}_{-}}(z)+C_{+} 1_{\mathbb{R}_{+}}(z)\right) d z .
$$

Note that the formal expression for $\mu_{\alpha, C_{-}, C_{+}}$coincides with that for the Lévy measure of an $\alpha$-stable distribution, although for $\alpha \in(2,2 /(1-2 H))$ an " $\alpha$-stable distribution" itself does not exist.

In contrast to (18), formula (65) contains explicitly the time parameter $t$. In addition, the polynomial "shape" of the expression in the right-hand side of (65) depends on $\alpha$, i.e., on the "shape" of the tails of the Lévy measure $\mu$.

Example 2. When the Lévy measure $\mu$ is "extremely heavy-tailed" in the sense explained above, the function $\mathfrak{m}$ may fail to belong to the class $\mathcal{L} d$ at all. Consider the measure

$$
\mu(d x)=\delta_{-1}(d x)+\sum_{k \geq 0} 2^{k-2 k /(1-2 H)} \delta_{2^{k}}(d y) .
$$

Then $\mu\left(\mathbb{R}_{-}\right)>0$, (17) fails, whereas (5) is satisfied:

$$
\int_{\mathbb{R}}|y|^{2 /(3-2 H)} \mu(d y)=1+\sum_{k \geq 0} 2^{2 k /(3-2 H)} 2^{k-2 k /(1-2 H)}=1+\sum_{k \geq 0} 2^{-k \cdot \frac{1+4 H(2-H)}{(3-2 H)(1-2 H)}}<\infty .
$$

Using (59), we can write $\mathfrak{m}(r)$ explicitly:

$$
\mathfrak{m}(r)=-\ell(-r)+c_{H} r^{-(3-2 H) /(1-2 H)} \sum_{k: 2^{k} \leq r \Gamma(H+1 / 2)} 2^{k}, \quad r \geq 0,
$$

where $c_{H}$ is defined in (19). To shorten the notation, put $c:=(\Gamma(H+1 / 2))^{-1}$. We have for $r_{n}:=2^{n} c$ and $r_{n}^{\prime}:=\left(2^{n}-1\right) c$, respectively,

$$
\mathfrak{m}\left(r_{n}\right)=\mathfrak{m}\left(2^{n} c\right)=-\ell\left(-2^{n} c\right)+c_{H}\left(2^{n} c\right)^{-(3-2 H) /(1-2 H)} \sum_{k=0}^{n} 2^{k}
$$

and

$$
\mathfrak{m}\left(r_{n}^{\prime}\right)=\mathfrak{m}\left(\left(2^{n}-1\right) c\right)=-\ell\left(-\left(2^{n}-1\right) c\right)+c_{H}\left(\left(2^{n}-1\right) c\right)^{-(3-2 H) /(1-2 H)} \sum_{k=0}^{n-1} 2^{k} .
$$

Note that

$$
\ell\left(-\left(2^{n}-1\right) c\right) \sim \ell\left(-2^{n} c\right) \sim-c_{H}\left(-2^{n} c\right)^{-(3-2 H) /(1-2 H)} \quad \text { and } \quad \sum_{k=0}^{n} 2^{k} \sim 2^{n+1}
$$

as $n \rightarrow \infty$. Therefore,

$$
\lim _{n \rightarrow \infty} \frac{\mathfrak{m}\left(\left(2^{n}-1\right) c\right)}{\mathfrak{m}\left(2^{n} c\right)}=\frac{1}{2}
$$

and $\mathfrak{m} \notin \mathcal{L} d$. 
From these two examples one can see that in the "extremely heavy-tailed" case the asymptotic behaviour of the distribution density of $Z_{t}^{H}$ is more sensitive with respect to the behaviour of the "tails" of the Lévy measure $\mu$ than in the case where the integrability condition (17) holds true. If these tails are regularly varying, then (16) holds true with the right hand side depending both on $t$ and on the "shape" of the "tails" of $\mu$. On the other hand, when the "tails" of $\mu$ are both "heavy" and "irregular", the function m may fail to belong to the class $\mathcal{L} d$, which means that we can not apply Theorem 2 at all.

\section{Appendix I: Existence of integral (1)}

Proposition 7. Let $0<H<1, H \neq 1 / 2$. Then the integral (1) is well defined for every $t \in \mathbb{R}$ if, and only if, the Lévy measure $\mu$ satisfies (5).

Proof. We consider the case $0<H<1 / 2$, the calculations in the case $1 / 2<H<1$ are analogous. We check the necessary and sufficient condition for the existence of (1) formulated in [23, Theorem 2.7]. In our case these conditions can be rewritten as

$$
\begin{gathered}
\int_{-\infty}^{1} f^{2}(s) d s<\infty, \\
I_{1}:=\int_{-\infty}^{1} \int_{\mathbb{R}}\left(1 \wedge|f(s) x|^{2}\right) \mu(d x) d s<\infty,
\end{gathered}
$$

and

$$
I_{2}:=\int_{-\infty}^{1}\left|\int_{\mathbb{R}}(\tau(f(s) x)-f(s) \tau(x)) \mu(d x)\right| d s<\infty
$$

where

$$
\tau(x)=\left\{\begin{array}{lll}
x, & \text { if } & |x| \leq 1 \\
\frac{x}{|x|}, & \text { if } & |x|>1
\end{array}\right.
$$

and $f(s):=f(1, s)$. Clearly, (66) is satisfied. We show that a) (67) and (5) are equivalent, b) (68) follows from (5).

a) Split

$$
I_{1}=I_{11}+I_{12}+I_{13}+I_{14}
$$

where

$$
\begin{aligned}
& I_{11}:=\int_{-\infty}^{-1} \int_{|u f(s)| \leq 1} \ldots, \quad I_{12}:=\int_{-1}^{1} \int_{|u f(s)| \leq 1} \ldots \\
& I_{13}:=\int_{-\infty}^{-1} \int_{|u f(s)|>1} \ldots, \quad I_{14}:=\int_{-1}^{1} \int_{|u f(s)|>1} \ldots
\end{aligned}
$$

and estimate the integrals $I_{1 i}, i=1, . ., 4$, separately.

Since $f(s) \sim-\frac{2 H-1}{2 \Gamma(H+1 / 2)}|s|^{H-3 / 2}$ as $s \rightarrow-\infty, f(s) \sim-\frac{1}{\Gamma(H+1 / 2)}|s|^{H-1 / 2}$, as $s \rightarrow 0-$, and $f(s)=\frac{1}{\Gamma(H+1 / 2)}(1-s)^{H-1 / 2}$ for $0 \leq s<1$, to check the finiteness of $I_{1}$ it is enough to substitute $f(s)$ in the regions $(-\infty,-1]$ and $(-1,0)$ with, respectively, $-|s|^{H-3 / 2}$ and $-|s|^{H-1 / 2}$, and to check the finiteness of the integrals $\tilde{I}_{11}:=\int_{1}^{\infty} \int_{|x| \leq s^{3 / 2-H}} \ldots, \tilde{I}_{12}:=$ $\int_{0}^{1} \int_{|x| \leq s^{1 / 2-H}} \cdots, \tilde{I}_{13}:=\int_{1}^{\infty} \int_{|x|>s^{3 / 2-H}} \ldots$, and $\tilde{I}_{14}:=\int_{0}^{1} \int_{|x|>s^{1 / 2-H}} \cdots$ 
We get:

$$
\begin{aligned}
& \tilde{I}_{11}= \int_{1}^{\infty} \frac{1}{s^{3-2 H}} \int_{|x| \leq s^{3 / 2-H}}|x|^{2} \mu(d x) d s \\
&= \int_{1}^{\infty} \frac{1}{s^{3-2 H}} \int_{|x| \leq 1}|x|^{2} \mu(d x) d s+\int_{1}^{\infty} \frac{1}{s^{3-2 H}} \int_{1<|x| \leq s^{3 / 2-H}}|x|^{2} \mu(d x) d s \\
&= \frac{1}{2-2 H}\left(\int_{|x| \leq 1}|x|^{2} \mu(d x)+\int_{|x| \geq 1}|x|^{2 /(3-2 H)} \mu(d x)\right) ; \\
& \tilde{I}_{12}=\int_{0}^{1} s^{2 H-1} \int_{|x| \leq s^{1 / 2-H}}|x|^{2} \mu(d x) d s \leq \frac{1}{2 H} \int_{|x| \leq 1}|x|^{2} \mu(d x) ; \\
& \tilde{I}_{13}=\int_{1}^{\infty} \int_{|x| \geq s^{3 / 2-H}} \mu(d x) d s=\int_{|x| \geq 1}\left(|x|^{2 /(3-2 H)}-1\right) \mu(d x) ; \\
& \tilde{I}_{14}=\int_{0}^{1} \int_{|x| \geq s^{1 / 2-H}} \mu(d x) d s=\int_{|x| \leq 1}|x|^{2 /(1-2 H)} \mu(d x)+\int_{|x| \geq 1} \mu(d x) .
\end{aligned}
$$

Therefore, $I<\infty$ if and only if (5) holds true.

b) Split $I_{2}:=I_{21}+I_{22}$, where $I_{21}:=\int_{-\infty}^{1} \int_{|u f(s)| \leq 1} \cdots$ and $I_{22}:=\int_{-\infty}^{1} \int_{|u f(s)| \geq 1} \cdots$

Observe that

$$
\int_{|x| \leq 1 /|u|}(u x-u \tau(x)) \mu(d x)=\int_{1 \leq|x| \leq 1 /|u|}\left(u x-\frac{u x}{|x|}\right) \mu(d x) \leq 2 \int_{1 \leq|x| \leq 1 /|u|}|u x| \mu(d x) .
$$

Then

$$
I_{21} \leq 2 \int_{-\infty}^{-1} \int_{1 \leq|x| \leq 1 /|f(s)|}|f(s) x| \mu(d x) d s .
$$

To estimate the right-hand side of (71) it is enough to estimate

$$
\tilde{I}_{21}:=\int_{1}^{\infty} \int_{1 \leq|x| \leq s^{3 / 2-H}} \frac{|x|}{s^{3 / 2-H}} \mu(d x) d s=\frac{2}{1-2 H} \int_{|x| \geq 1}|x|^{2 /(3-2 H)} \mu(d x) .
$$

Thus, (5) implies the finiteness of $\tilde{I}_{21}$, and, consequently, of (71).

To estimate $I_{22}$ observe that

$$
\begin{aligned}
\int_{|x| \geq 1 /|u|} & \left(\frac{x u}{|x u|}-u \tau(x)\right) \mu(d x) \\
& =\int_{|x| \geq \max (1 /|u|, 1)}\left(\frac{x u}{|x u|}-u \frac{x}{|x|}\right) \mu(d x)+\int_{1 /|u| \leq|x| \leq 1}\left(\frac{x u}{|x u|}-u x\right) \mu(d x) .
\end{aligned}
$$


Then

$$
\begin{aligned}
& I_{22} \leq 2\left(\int_{-\infty}^{-1} \int_{|x| \geq 1 /|f(s)|} \mu(d x) d s+\int_{-1}^{1} \int_{|x| \geq 1}|f(s)| \mu(d x) d s\right. \\
& \left.+\int_{-1}^{1} \int_{1 /|f(s)| \leq|x| \leq 1}|f(s)| \mu(d x) d s\right) \\
& \leq C_{1}\left(\int_{1}^{\infty} \int_{|x| \geq s^{3 / 2-H}} \mu(d x) d s+\int_{-1}^{1}|f(s)| d s \int_{|x| \geq 1} \mu(d x)\right. \\
& \left.+\int_{0}^{1} \int_{s^{1 / 2-H} \leq|x| \leq 1} s^{H-1 / 2} \mu(d x) d s\right) \\
& \leq C_{2}\left(\int_{|x| \geq 1}|x|^{2 /(3-2 H)} \mu(d x)+\int_{|x| \geq 1} \mu(d x)+\int_{|x| \leq 1}|x|^{(1+2 H) /(1-2 H)} \mu(d x)\right),
\end{aligned}
$$

and the finiteness of the right-hand side is implied by (5).

\section{Appendix II: Existence of the distribution density}

Proposition 8. Let $0<H<1, H \neq 1 / 2$. Then, under (5) and our standing assumption (6), the integral (1) possesses for any $t \neq 0$ the distribution density $p_{t} \in C_{b}^{\infty}(\mathbb{R})$.

Remark 3. In the non-Markov case $H \neq 1 / 2$, the kernel $f(t, s)$ provides a strong "smoothifying" effect in the sense that the weakest possible non-degeneracy assumption (6) is already sufficient for the integral (1) to possess a smooth distribution density. We refer to [18], Section 3, for the detailed discussion of various forms of the "smoothifying" effect for Lévy driven stochastic integrals with deterministic kernels.

For the proof we use the following statement, see [18, Lemma 3.3].

Proposition 9. a) For a positive function $h(s)$ having a continuous non-zero derivative on some interval $[a, b] \subset \mathbb{R}$, one has

$$
\int_{a}^{b}(1-\cos (h(s) x)) d s \geq c\left(x^{2} \wedge 1\right) .
$$

b) For a positive convex on $(-\infty, b) \subset \mathbb{R}$ function $h(s)$, satisfying

$$
\lim _{s \rightarrow-\infty} e^{-\gamma s} h(s)=+\infty \quad \text { for all } \quad \gamma>0
$$

one has

$$
\int_{-\infty}^{b}(1-\cos (x h(s))) d s \geq c \ln |x|
$$

for all $c>0$ and $|x|$ big enough.

Proof of Proposition 8. Recall (cf. (7)) that the characteristic function of $Z_{t}^{H}$ is of the form $\phi(t, z)=e^{\Psi(t,-z)}$. For a fixed $t$, the function $h(s)=-t^{H-1 / 2} f(s)$ satisfies (73) with $b=0$. Since $\mu(\mathbb{R})>0$ (cf. (6)), there exists $q>0$ such that

$$
Q:=\max \{\mu((-\infty,-q]), \mu([q, \infty)\}>0 .
$$


Then using (74) for $|z|$ large enough we get

$$
-\operatorname{Re} \Psi(t,-z) \geq t \int_{-\infty}^{0} \int_{|u| \geq q}\left(1-\cos \left(t^{H-1 / 2} f(s) u z\right)\right) \mu(d u) d s \geq t c Q \ln \left|q t^{H-1 / 2} z\right| .
$$

Since $c>0$ is arbitrary, the function $|z|^{n}|\phi(t, z)|=e^{\operatorname{Re} \Psi(t,-z)+n \ln |z|}$ is integrable in $z$ for any $n \geq 1$. Therefore the density $p_{t}$ is well defined and belongs to $C_{b}^{\infty}$ as the inverse Fourier transform of $\phi(t, z)$ :

$$
p_{t}(x)=\frac{1}{2 \pi} \int_{\mathbb{R}} e^{-i z x} \phi(t, z) d z .
$$

Remark 4. Literally the same argument implies that, if the truncation level $\lambda>0$ is chosen small enough, then $|z|^{n}\left|\phi_{1}(t, z)\right|=e^{\operatorname{Re} \Psi_{1}(t,-z)+n \ln |z|}$ is integrable in $z$ for any $a>0$, $t>0$, see (39). This implies the existence of $\tilde{p}_{t}(x)$, see $(42)$.

Acknowledgement. The authors thank the referee for helpful remarks, and gratefully acknowledge the DFG Grant Schi 419/8-1. The first-named author also gratefully acknowledges the Scholarship of the President of Ukraine for young scientists (2011-2013).

\section{References}

[1] Basse, A., Pedersen, J.: Lévy driven moving averages and semimartingales. Stochastic Processes Appl. 119, 2970-2991 (2009).

[2] Benassi, A., Cohen, S., Istas, J.: On roughness indices for fractional fields. Bernoulli 10(2), 357-373 (2004)

[3] Bender, C., Marquardt, T.: Integrating volatility clustering into exponential Lévy models. J. Appl. Probab. 46(3), 609-628 (2009)

[4] Bender, C., Lindner, A., Schicks, M.: Finite Variation of Fractional Lévy Processes. J. Theor. Probab. 25(2), 594-612 (2012)

[5] Bender, C., Sottinen, T., Valkeila, E.: Pricing by hedging and no-arbitrage beyond semimartingales. Finance Stoch. 12(4), 441-468 (2008)

[6] Burnecki, K., Weron. A.: Fractional Lévy stable motion can model subdiffusive dynamics. Phys. Rev. E82, 021130 (2010)

[7] Calvo, I., Sánchez, R., Carreras, A.: Fractional Lévy motion through paths integrals. J. Phys. A 42, 055003 (2009)

[8] Chechkin, A. V., Gonchar, V. Yu.: A Model for Persistent Lévy Motion. Physica A277, 312-326 (2000)

[9] Copson, E. T.: Asymptotic expansions. Cambridge Uni. Press, Cambridge (1965) 
[10] Dimakis, A. G., Maragos, P.: Phase-Modulated Resonances Modeled as Self-Similar Processes With Application to Turbulent Sounds. IEEE Trans. Signal Process. 53(11) (2005)

[11] Eliazar, I. I., Shlesinger, M. F.: Langevin unification of fraction motions. J. Phys. A45, 162002 (2012)

[12] Feller, W.: An Introduction to Probability Theory and Its Applications. Vol. II. Second edition John Wiley \& Sons, Inc., New York-London-Sydney (1971)

[13] J.-S. Gagnon, S. Lovejoy, D. Schertzer. Multifractal earth topography. Nonlinear processes in geophysics. 13 (5), 541-570 (2006)

[14] Hartman, P., Wintner, A.: On the infinitesimal generators of integral convolutions. Am. J. Math. 64, 273-298 (1942)

[15] Huillet, T.: Fractional Lévy motions and related processes. J. Phys. A 32, 7225 (1999)

[16] Kallenberg, O.: Splitting at backward times in regenerative sets. Ann. Prob. 9(5), 781-799 (1981)

[17] Klüppelberg, C.: Subexponential Distributions and Characterizations of Related Classes. Probab. Th. Rel. Fields. 82, 259-269 (1989)

[18] Knopova, V., Kulik, A.: Exact asymptotic for distribution densities of Lévy functionals. Electronic J. Probab. 16, 1394-1433 (2011)

[19] Kogon, S. M. Manolakis, D. G.: Signal modeling with self-similar $\alpha$-stable processes: the fractional levy stable motion model. IEEE Trans. Signal Process. 44(4), 10061010 (1996)

[20] Laskin, N., Lambadaris, I., Harmantzis, F., Devetsikiotis, M.: Fractional Lévy Motion and its Application to Traffic Modeling. Computer Networks (Special Issue on Long-Range Dependent Traffic Engineering) 40(3), 363-375 (2002)

[21] Marquardt, T.: Fractional Lévy processes with an application to long memory moving average processes. Bernoulli 12(6), 1099-1126 (2006)

[22] Painter, S., Patterson. L.: Fractional Lévy Motion as a Model for Spatial Variability in Sedimentary rock. Geophys. Res. Let. 21(25), 2857-2860 (1994)

[23] Rajput, B.S., Rosinski, J.: Spectral representations of infinitely divisible processes. Probab. Th. Rel. F. 82, 451-487 (1989)

[24] Samorodnitsky, G., Taqqu, M. S.: Stable Non-Gaussian Random Processes. Chapman Hall, New York (1994)

[25] Tikanmäki, H. Mishura, Yu.: Fractional Lévy processes as a result of compact interval integral transformation. Stoch. Anal. Appl. 29, 1081-1101 (2011) 
[26] Watkins, N. W., Credgington, D., Hnat, B., Chapman, S. C., Freeman, M. P., Greenhough. J.: Towards Synthesis of Solar Wind and Geomagnetic Scaling Exponents: A Fractional Lévy Motion Model Space Sci. Rev. 121 (1-4), 271-284 (2005)

[27] Weron, A., Burnecki, K., Mercik, Sz., Weron, K.: Complete description of all selfsimilar models driven by Lévy stable noise. Phys. Rev. E71 (2005), 016113 (2005) 